\title{
Retinal Sensitivity Is Reduced in Patients With Obstructive Sleep Apnea
}

\author{
Blanca Ferrandez, ${ }^{1}$ Antonio Ferreras, ${ }^{1,2}$ Pilar Calvo, ${ }^{1}$ Beatriz Abadia,${ }^{1}$ Paolo Fogagnolo, ${ }^{3}$ \\ Yaowu Wang, ${ }^{4}$ Jose M. Marin, ${ }^{5}$ and Michele Iester ${ }^{6}$ \\ ${ }^{1}$ Department of Ophthalmology, Miguel Servet University Hospital, Aragon Health Sciences Institute, Zaragoza, Spain \\ ${ }^{2}$ University of Zaragoza, Spain \\ ${ }^{3}$ G.B. Bietti Foundation-Istituto di Ricovero e Cura a Carattere Scientifico (IRCCS), Rome, Italy \\ ${ }^{4}$ Faculty of Medicine, University of Toronto, Canada \\ ${ }^{5}$ Department of Pneumology, Miguel Servet University Hospital, Zaragoza, Spain \\ ${ }^{6}$ University of Genoa, Genoa, Italy
}

Correspondence: Antonio Ferreras, Department of Ophthalmology, Miguel Servet University Hospital, Isabel la Catolica 1-3, 50009 Zaragoza, Spain;

aferreras@msn.com.

Submitted: March 18, 2014

Accepted: September 26, 2014

Citation: Ferrandez B, Ferreras A, Calvo P, et al. Retinal sensitivity is reduced in patients with obstructive sleep apnea. Invest Ophthalmol Vis Sci. 2014;55:7119-7125. DOI:

10.1167/iovs.14-14389
Purpose. To evaluate the outcomes of standard automated perimetry (SAP) in patients with obstructive sleep apnea (OSA).

Methods. Eighty OSA patients and 111 age-matched controls were consecutively and prospectively enrolled. One eye per subject was randomly selected. All participants underwent at least one reliable SAP (24-2 SITA Standard algorithm). The peripapillary retinal nerve fiber layer thickness (RNFL) was measured with spectral-domain optical coherence tomography (OCT). Patients with OSA were classified into three groups according to the apnea/hypopnea index: mild, moderate, or severe OSA. Parameters of SAP and OCT were compared between healthy controls and OSA patients. Correlation of apnea/hypopnea index with OCT and SAP measurements were calculated.

Results. Mean age, best-corrected visual acuity, and central corneal thickness were similar between groups. Intraocular pressure, however, was lower in the OSA group. Mean deviation of SAP was $-0.23 \pm 0.8 \mathrm{~dB}$ in the control group and $-1.74 \pm 2.8 \mathrm{~dB}$ in the OSA group $(P<$ 0.001). Thickness of RNFL measured with OCT did not differ significantly between groups. Patients with OSA showed reduced sensitivity at most points tested by white-on-white perimetry compared with healthy individuals. The threshold values were more depressed in the peripheral visual field. The apnea/hypopnea index was related to the SAP indices: Pearson correlations were -0.432 with mean deviation, 0.467 with pattern standard deviation, and -0.416 with the visual field index $(P<0.001)$.

Conclusions. Patients with OSA exhibited reduced retinal sensitivity measured with SAP compared with healthy controls.

Keywords: obstructive sleep apnea, visual field, optical coherence tomography, retinal nerve fiber layer, optic disc
$\mathrm{O}$ bstructive sleep apnea (OSA) syndrome is a disorder characterized by recurrent partial (hypopnea) or complete (apnea) cessation of breathing during sleep. When the apnea/ hypopnea index (AHI) is at least five events per hour, OSA is considered a clinical entity. Obstructive sleep apnea has a reported prevalence of more than 20\% in the general population $^{1,2}$ and is associated with cardiovascular, neurologic, and endocrine disease..$^{3-10}$ Sympathetic activation, oxidative stress, and systemic inflammation are the main intermediary mechanisms associated with sleep apnea and intermittent hypoxia. $^{11}$

Retinal ganglion cells provide a peripheral but accessible window to central nervous system neurons that could be subject to neuronal damage in association with OSA. Hypoxia and oxidative stress are risk factors for neuronal death in retinal diseases such as diabetic retinopathy and glaucoma. ${ }^{12,13}$ This neuronal injury may result in thinning of the retinal nerve fiber layer (RNFL) or reduced visual field (VF) sensitivity.
Noninvasive imaging technologies, such as spectral domain optical coherence tomography (OCT), provide quantitative and reproducible measurements for assessing RNFL thickness. Numerous studies have confirmed the ability of OCT to detect and monitor glaucoma, ${ }^{14-19}$ as well as to diagnose and followup other disorders that affect the optic nerve head (ONH), such as Parkinson disease, multiple sclerosis, and Alzheimer disease. $^{20-25}$

The purpose of the present study was to compare the VF and OCT parameters between healthy individuals and OSA patients.

\section{Materials ANd Methods}

The study design adhered to the tenets of the Declaration of Helsinki and was approved by the Clinical Research Ethics Committee of Aragon. Healthy eyes were recruited from the hospital staff or relatives, and patients referred for refraction 
TABLE 1. Clinical Characteristics of the Study Population

\begin{tabular}{lrrrrrrr}
\hline & \multicolumn{2}{c}{ Control } & & \multicolumn{2}{c}{ OSA } & \\
\cline { 2 - 3 } & Mean & SD & & Mean & SD & $P^{*}$ \\
\hline Age, y & 50.78 & 9.46 & & 48.83 & 11.52 & 0.20 \\
BCVA, logMAR & 0.04 & 0.05 & & 0.02 & 0.05 & 0.02 \\
Pachymetry, $\mu \mathrm{m}$ & 551.66 & 35.60 & & 560.01 & 30.12 & 0.10 \\
IOP, mm Hg & 17.09 & 2.46 & & 13.99 & 2.60 & $<\mathbf{0 . 0 0 1}$ \\
MD SAP, dB & -0.23 & 0.82 & & -1.74 & 2.77 & $<\mathbf{0 . 0 0 1}$ \\
PSD SAP & 1.42 & 0.23 & & 1.98 & 1.05 & $<\mathbf{0 . 0 0 1}$ \\
VFI SAP & 99.52 & 0.70 & & 97.51 & 4.83 & $<\mathbf{0 . 0 0 1}$ \\
SAP test duration, s & 319.90 & 39.31 & & 331.40 & 45.52 & 0.06 \\
Fixation losses SAP, \% & 3.79 & 3.38 & & 4.23 & 4.17 & 0.42 \\
False positive rate SAP, \% & 1.07 & 1.25 & & 1.05 & 0.98 & 0.90 \\
False negative rate SAP, \% & 0.61 & 0.98 & & 0.72 & 0.95 & 0.44 \\
Systolic pressure, mm Hg & 118.98 & 12.32 & & 124.11 & & 14.76 & 0.01 \\
Diastolic pressure, mm Hg & 75.83 & 8.82 & & 78.74 & 9.78 & 0.03 \\
BMI, kg/m ${ }^{2}$ & 24.25 & 4.78 & & 27.82 & 7.21 & $<\mathbf{0 . 0 0 1}$ \\
$n$ & 111 & & 80 & & \\
\hline
\end{tabular}

* Student's $t$-test. Significant differences $(P \leq 0.001)$ are highlighted in bold font.

that underwent routine examination without abnormal ocular findings, obesity, or history of symptoms related to OSA (sleepiness, fatigue, snoring, adenoid facies, etc.). The group with OSA was selected from among patients at the Respiratory Unit of the Miguel Servet University Hospital in Zaragoza, Spain. The group with OSA had no significant ophthalmic disease and was not followed more frequently than the healthy group. All individuals who met the inclusion criteria and provided written informed consent were consecutively and prospectively enrolled from January 2011 to December 2012.

All patients with OSA underwent an overnight sleep study in the hospital for evaluation and diagnosis of OSA syndrome. The diagnosis of OSA was based on a polysomnographic evaluation comprising electroencephalography, electromyography, electrocardiography, respiratory movements, and pulse oximetry measurements. These tests were performed over a minimum of 6 hours in the electrophysiology department of our hospital. Dual respiratory inductance plethysmography belt signals (thorax and abdomen) were used for scoring apnea and hypopnea. Sleep and respiratory events in each patient were recorded according to standard criteria. ${ }^{26}$ An apnea event was defined as a decrease in the peak signal excursion $\geq 90 \%$ for at least 10 seconds. A hypopnea event was defined as a decrease in the peak signal excursion $\geq 30 \%$ (and $<90 \%$ ) from the pre-event baseline for at least 10 seconds, and $\geq 3 \%$ oxygen desaturation from the pre-event baseline, or an event associated with arousal. Individuals with an AHI of at least five events/h were considered to have OSA. Severity of OSA was classified according to the AHI as follows: mild OSA when the AHI was between 5 and 14 events/h, moderate OSA when the AHI ranged from 15 to 19 events/h, and severe OSA when the AHI was higher than 19 events/h.

Inclusion criteria were best-corrected visual acuity of 20/40 or better, refractive error within \pm 5.00 diopters (D) equivalent sphere, and $\pm 2 \mathrm{D}$ astigmatism; transparent ocular media (nuclear color and opalescence, cortical or posterior subcapsular lens opacity $<1$ ) according to the Lens Opacities Classification System III system ${ }^{27}$; and open anterior chamber angle. Exclusion criteria were previous intraocular surgery, diabetes, history of any ocular or neurologic disease, and any treatment with oxygen therapy or noninvasive mechanical ventilation. Patients with glaucomatous standard automated perimetry (SAP) defects, glaucomatous optic disc morphology, or intraocular pressure (IOP) $>20 \mathrm{~mm} \mathrm{Hg}$ were also excluded from the study. A total of 80 patients with OSA and 111 healthy subjects were included in the statistical analysis.

\section{Study Protocol}

All examinations were performed within 6 weeks of the subject's date of enrollment into the study. Participants underwent a comprehensive ophthalmologic examination: clinical history, best corrected visual acuity (BCVA), slit-lamp biomicroscopy of the anterior segment, gonioscopy, Goldmann applanation tonometry, central corneal ultrasonic pachymetry (OcuScan RxP; Alcon Laboratories, Inc., Irvine, CA, USA), and ophthalmoscopy of the posterior segment.

Two SAPs were performed using a Humphrey field analyzer (model 750i; Zeiss Humphrey Systems, Dublin, CA, USA), with the 24-2 SITA Standard strategy. Subjects completed the perimetry tests before undergoing any clinical examination or structural test, and each test was performed at least 3 days apart to avoid a fatigue effect. All participants had a reliable SAP (false positives and negatives $<15 \%$, fixation losses $<$ $20 \%)$ in the second attempt. The data from the last perimetry test were used for the statistical analysis to minimize the learning effect.

Peripapillary RNFL thickness was measured using the Optic Disc Cube $200 \times 200$ acquisition protocol of the OCT device (Cirrus HD, software version 6.2; Carl Zeiss Meditec, Dublin, CA, USA). This protocol scanned a $6 \times 6 \mathrm{~mm}^{2}$ area centered on the optic disc. A $3.46-\mathrm{mm}$ diameter circular scan, containing 256 A-scans, was automatically targeted around the optic disc to provide the RNFL thicknesses of the four quadrants and at each of the 12 clock-hour positions. Left eyes were converted to a right eye format. All images were obtained by the same experienced technician with a signal/strength ratio greater than $6 / 10$.

\section{Statistical Analysis}

Statistical analyses were performed using statistical software (IBM SPSS, version 21.0; IBM Corporation, Somers, NY, USA). When both eyes fulfilled the inclusion criteria, only one eye per subject was randomly selected for the study. All study variables had a normal distribution as verified with the Kolmogorov-Smirnov test (K-S of 1 sample). Demographics, SAP, and OCT parameters were compared between groups with Student's $t$-tests. The Bonferroni correction for multiple comparisons was applied, resulting in a significance level of $P$ $<0.002$. Pearson correlations were calculated between the $\mathrm{AHI}$ and VF indices.

\section{Results}

The clinical characteristics of the study sample are detailed in Table 1. A total of 111 healthy controls and 80 OSA patients were included in the statistical analysis. Mean age was $50.78 \pm$ 9.4 years in the control group and $48.8 \pm 11.5$ years in the OSA group $(P=0.19)$. Central corneal thickness was similar in both groups $(551.66 \pm 35.6 \mu \mathrm{m}$ in healthy subjects and 560.01 $\pm 30.1 \mu \mathrm{m}$ in OSA patients; $P=0.09$ ), but intraocular pressure was significantly lower in the OSA group $(17.09 \pm 2.5 \mathrm{~mm} \mathrm{Hg}$ in healthy subjects and $13.99 \pm 2.6 \mathrm{~mm} \mathrm{Hg}$ in OSA patients; $P$ $<0.001)$.

Main SAP indices differed significantly between the groups. Mean deviation (MD) was lower in the OSA group $(-0.23 \pm 0.8$ dB vs. $-1.74 \pm 2.8 \mathrm{~dB} ; P<0.001)$, pattern standard deviation (PSD) was higher in the OSA group $(1.42 \pm 0.2$ vs. $1.98 \pm 1.1$; $P<0.001)$, and the visual field index (VFI) was lower in the 
TABLE 2. Differences in Sensitivity Threshold Values (dB) of Tested VF Points in SAP Between Control and OSA Groups

\begin{tabular}{|c|c|c|c|c|c|}
\hline & \multicolumn{2}{|c|}{ Control Group } & \multicolumn{2}{|c|}{ OSA Group } & \multirow[b]{2}{*}{$\boldsymbol{P}^{*}$} \\
\hline & Mean & SD & Mean & SD & \\
\hline SAP 1 & 27.24 & 2.22 & 25.59 & 3.79 & $<0.001$ \\
\hline SAP 2 & 28.06 & 2.16 & 26.04 & 4.48 & $<0.001$ \\
\hline SAP 3 & 27.33 & 1.98 & 25.11 & 4.83 & $<0.001$ \\
\hline SAP 4 & 27.18 & 2.63 & 24.78 & 5.34 & $<0.001$ \\
\hline SAP 5 & 28.62 & 2.24 & 27.04 & 3.40 & $<0.001$ \\
\hline SAP 6 & 29.70 & 1.59 & 28.26 & 2.76 & $<0.001$ \\
\hline SAP 7 & 29.71 & 1.44 & 28.16 & 3.09 & $<0.001$ \\
\hline SAP 8 & 29.27 & 1.61 & 26.99 & 4.72 & $<0.001$ \\
\hline SAP 9 & 29.39 & 2.01 & 28.08 & 3.17 & 0.001 \\
\hline SAP 10 & 29.13 & 2.27 & 26.75 & 5.33 & $<0.001$ \\
\hline SAP 11 & 28.46 & 2.26 & 26.41 & 4.40 & $<0.001$ \\
\hline SAP 12 & 30.43 & 1.42 & 29.18 & 2.60 & $<0.001$ \\
\hline SAP 13 & 31.61 & 1.27 & 29.93 & 4.54 & $<0.001$ \\
\hline SAP 14 & 31.51 & 1.40 & 30.51 & 2.89 & 0.002 \\
\hline SAP 15 & 31.05 & 1.37 & 29.60 & 3.06 & $<0.001$ \\
\hline SAP 16 & 30.32 & 1.65 & 28.85 & 3.31 & $<0.001$ \\
\hline SAP 17 & 30.20 & 1.92 & 28.68 & 3.81 & $<0.001$ \\
\hline SAP 18 & 28.68 & 2.22 & 26.69 & 4.93 & $<0.001$ \\
\hline SAP 19 & 26.86 & 2.61 & 25.06 & 5.16 & 0.002 \\
\hline SAP 20 & 29.00 & 1.68 & 27.53 & 3.73 & $<0.001$ \\
\hline SAP 21 & 31.30 & 1.35 & 29.90 & 3.15 & $<0.001$ \\
\hline SAP 22 & 32.38 & 1.14 & 30.74 & 4.00 & $<0.001$ \\
\hline SAP 23 & 32.68 & 1.24 & 31.38 & 3.34 & $<0.001$ \\
\hline SAP 24 & 32.42 & 1.35 & 30.90 & 3.43 & $<0.001$ \\
\hline SAP 25 & 31.41 & 1.52 & 30.09 & 3.44 & $<0.001$ \\
\hline SAP 26 & 29.84 & 2.03 & 27.64 & 4.72 & $<0.001$ \\
\hline SAP 27 & 26.89 & 2.66 & 24.81 & 5.71 & 0.001 \\
\hline SAP 28 & 29.38 & 1.68 & 27.61 & 4.04 & $<0.001$ \\
\hline SAP 29 & 31.60 & 1.47 & 30.36 & 2.84 & $<0.001$ \\
\hline SAP 30 & 32.50 & 1.15 & 31.23 & 3.05 & 0.001 \\
\hline SAP 31 & 32.66 & 1.27 & 31.87 & 2.39 & 0.004 \\
\hline SAP 32 & 32.66 & 1.39 & 31.66 & 2.66 & 0.001 \\
\hline SAP 33 & 31.76 & 1.49 & 30.41 & 2.76 & $<\mathbf{0 . 0 0 1}$ \\
\hline SAP 34 & 30.25 & 2.04 & 28.64 & 3.77 & $<0.001$ \\
\hline SAP 35 & 28.82 & 1.96 & 27.33 & 3.65 & $<0.001$ \\
\hline SAP 36 & 30.75 & 1.44 & 29.70 & 3.23 & 0.003 \\
\hline SAP 37 & 32.22 & 1.34 & 31.26 & 2.87 & 0.002 \\
\hline SAP 38 & 31.91 & 1.33 & 30.91 & 2.52 & $<0.001$ \\
\hline SAP 39 & 32.07 & 1.20 & 31.00 & 2.77 & $<0.001$ \\
\hline SAP 40 & 31.61 & 1.79 & 30.68 & 3.06 & 0.009 \\
\hline SAP 41 & 30.68 & 1.72 & 29.83 & 3.33 & 0.02 \\
\hline SAP 42 & 29.68 & 2.09 & 28.13 & 4.12 & 0.001 \\
\hline SAP 43 & 30.05 & 1.97 & 28.49 & 3.80 & $<0.001$ \\
\hline SAP 44 & 30.62 & 1.43 & 29.68 & 2.84 & 0.003 \\
\hline SAP 45 & 30.95 & 1.57 & 29.54 & 3.37 & $<\mathbf{0 . 0 0 1}$ \\
\hline SAP 46 & 30.77 & 1.61 & 29.53 & 3.15 & $<0.001$ \\
\hline SAP 47 & 30.93 & 1.49 & 29.85 & 3.45 & 0.004 \\
\hline SAP 48 & 30.92 & 1.69 & 28.85 & 4.39 & $<0.001$ \\
\hline SAP 49 & 28.92 & 2.20 & 27.16 & 4.25 & $<0.001$ \\
\hline SAP 50 & 29.16 & 1.95 & 27.31 & 5.16 & 0.001 \\
\hline SAP 51 & 29.93 & 1.88 & 28.14 & 4.20 & $<0.001$ \\
\hline SAP 52 & 30.00 & 2.05 & 28.10 & 4.90 & $<0.001$ \\
\hline
\end{tabular}

* Student's $t$-test. Significant differences $(P \leq 0.001)$ are highlighted in bold font.

OSA patients $(99.52 \pm 0.7$ vs. $97.51 \pm 1.1 ; P<0.001)$ Reliability indices of SAP and test time were similar between groups. Systolic and diastolic blood pressure did not differ between groups, but body mass index (BMI) was higher in the OSA group.
Table 2 shows the VF threshold values at each of the $52 \mathrm{VF}$ points tested by the grid of the 24-2 SITA Standard strategy (Fig. 1). Most of these points were depressed in OSA patients compared with healthy controls (Figs. 2, 3). The measurements of OCT revealed that the two groups were not different in peripapillary RNFL thickness at each of the 12 clock-hour positions, in the four quadrants, or in the average thickness (Table 3). Moreover, OSA patients grade 2 (moderate) and grade 3 (severe) combined or grade 3 alone had RNFL thicknesses similar to those in the control group (Table 4).

The VF indices of the SAP correlated moderately with the AHI: the Pearson correlation coefficients between AHI and MD, PSD, and VFI were $-0.432,0.467$, and -0.416 , respectively $(P$ $<0.001)$.

\section{Discussion}

The pathogenesis of neurodegeneration in OSA syndrome is unclear. Intermittent upper airway obstruction in OSA patients during sleep can increase carbon dioxide levels while decreasing oxygen levels, thereby reducing the oxygen saturation during a variable period of sleep. These alterations induce oxidative stress, and lead to increased vascular resistance and endothelial dysfunction. Hypoxia and hypercapnia episodes cause autonomic, hemodynamic, and neurorendocrine changes. ${ }^{28,29}$ Obstructive sleep apnea syndrome is associated with neurovascular disease, cardiovascular disease, arterial hypertension, pulmonary hypertension, arrhythmia, and other systemic disorders. ${ }^{30}$

The oxygenation-reoxygenation process in OSA syndrome can compromise normal vascularization and perfusion of the ONH similar to glaucoma, based on the vascular theory proposed by Anderson. ${ }^{31}$ This theory suggests that the damage to the $\mathrm{ONH}$ in glaucoma is caused, at least in part, by persistent or temporary ischemia. Failure of the blood supply from the posterior ciliary arteries to the short posterior ciliary arteries could lead to nonperfusion of the anterior part of the ONH and neuronal ischemia. The affected $\mathrm{ONH}$ microcirculation would interfere with axonal nutrition and axoplasmic flow. Calvo et al. ${ }^{32}$ reported that abnormal retrobulbar blood flow velocities measured by color Doppler ultrasound may be a risk factor for conversion to glaucoma. On the other hand, Lin et al. ${ }^{33}$ recently suggested that OSA is associated with an increased risk of a subsequent diagnosis of open-angle glaucoma during a 5-year follow-up period after the diagnosis of OSA.

We hypothesized that these vascular phenomena could affect VF and ONH morphology in OSA patients and that such changes could be measured by SAP and OCT, respectively. Retinal sensitivity was reduced in OSA patients compared with the control group, although we detected no significant differences in the RNFL thickness measurements. The topographic correspondence between VF regions and RNFL thickness in humans is moderate in glaucoma patients due to the high variability of normal human ONH morphology and the intertest variability of SAP. ${ }^{34-36}$ The agreement between the SAP and OCT results in OSA patients is expected to be similar to that in healthy individuals. ${ }^{34-38}$

Previous studies using different imaging techniques to measure RNFL thickness in OSA patients described contradictory results. ${ }^{39-44}$ Some studies reported decreased RNFL thickness in OSA patients, in contrast to the present results, ${ }^{39,40,42}$ whereas others found no correlation between RNFL thickness and AHI. ${ }^{42}$ Still others observed a significant decrease in the RNFL thickness in patients with severe OSA. ${ }^{44}$ All of these findings, however, were based on different imaging technologies than used in the present study. We measured the RNFL thickness with a spectral-domain OCT (Cirrus), whereas 


\section{OD}

\begin{tabular}{ccccc|cccc} 
& & & 1 & 2 & 3 & 4 & & \\
& & 5 & 6 & 7 & 8 & 9 & 10 & \\
& 11 & 12 & 13 & 14 & 15 & 16 & 17 & 18 \\
19 & 20 & 21 & 22 & 23 & 24 & 25 & & 26 \\
\hline 27 & 28 & 29 & 30 & 31 & 32 & 33 & & 34 \\
& 35 & 36 & 37 & 38 & 39 & 40 & 41 & 42 \\
& & 43 & 44 & 45 & 46 & 47 & 48 & \\
& & & 49 & 50 & 51 & 52 & &
\end{tabular}

OS

\begin{tabular}{cccc|ccccc} 
& & 4 & 3 & 2 & 1 & & & \\
& 10 & 9 & 8 & 7 & 6 & 5 & & \\
18 & 17 & 16 & 15 & 14 & 13 & 12 & 11 & \\
26 & & 25 & 24 & 23 & 22 & 21 & 20 & 19 \\
\hline 34 & & 33 & 32 & 31 & 30 & 29 & 28 & 27 \\
42 & 41 & 40 & 39 & 38 & 37 & 36 & 35 & \\
& 48 & 47 & 46 & 45 & 44 & 43 & & \\
& & 52 & 51 & 50 & 49 & & &
\end{tabular}

FiguRE 1. Grid of visual field points tested by the 24-2 SITA Standard algorithm of SAP. These locations were numbered as shown based on the side of the eye.

time-domain OCT $^{40,41,43}$ or scanning laser polarimetry ${ }^{39,42}$ were used in the previous studies.

If outcomes of VF or OCT differed between groups, these parameters could potentially be used as biomarkers for neuronal damage in OSA patients. Although we detected no changes in the peripapillary RNFL thickness measured by OCT, there were differences in the retinal sensitivity and VF indices. Patients with OSA exhibited diffuse decreased retinal sensitivity without localized VF defects typical of other pathologies such as glaucoma. This generalized reduction of the threshold values, particularly in the peripheral VF, could be explained by diffuse vascular damage to the retinal ganglion cells, causing axonal loss and VF depression. This injury to the retinal ganglion cells may lead to dysfunction of the visual pathway that could be identified by a functional test, such as SAP, while the anatomy is relatively unaffected and therefore undetected by a structural test, such as OCT. Moreover, OSA patients (especially those with more advanced disease) may have experienced at least one apnea/hypopnea event during the VF examination, which could decrease attention and impair testing ability, resulting in reduced retinal sensitivity, even when the SAP reliability criteria were maintained within normal limits.

Quantitative variables MD, PSD, and VFI could be used to monitor the progression of the disease, treatment with oxygen therapy, or noninvasive mechanical ventilation. ${ }^{45}$ The main criterion to begin treatment with continuous positive airway pressure (CPAP) is a graduated severity of OSA syndrome (moderate and severe). Thus, any changes detected in the VF could help to support the decision to begin CPAP in OSA patients.

Huseyinoglu et al. ${ }^{44}$ also reported worse MD and PSD (Octopus perimetry) in OSA subgroups compared to a control group. Lin et al., ${ }^{40}$ however, found no differences in the MD of SAP (30-2 strategy) between OSA patients and healthy controls. Different study designs and populations, inclusion and exclusion criteria, tests performed, and the level of OSA damage make it difficult to compare the results among several studies.

A limitation of the present study was that the control group did not undergo an overnight sleep study and we assumed that they had no apnea/hypopnea events per hour. Ideally, they
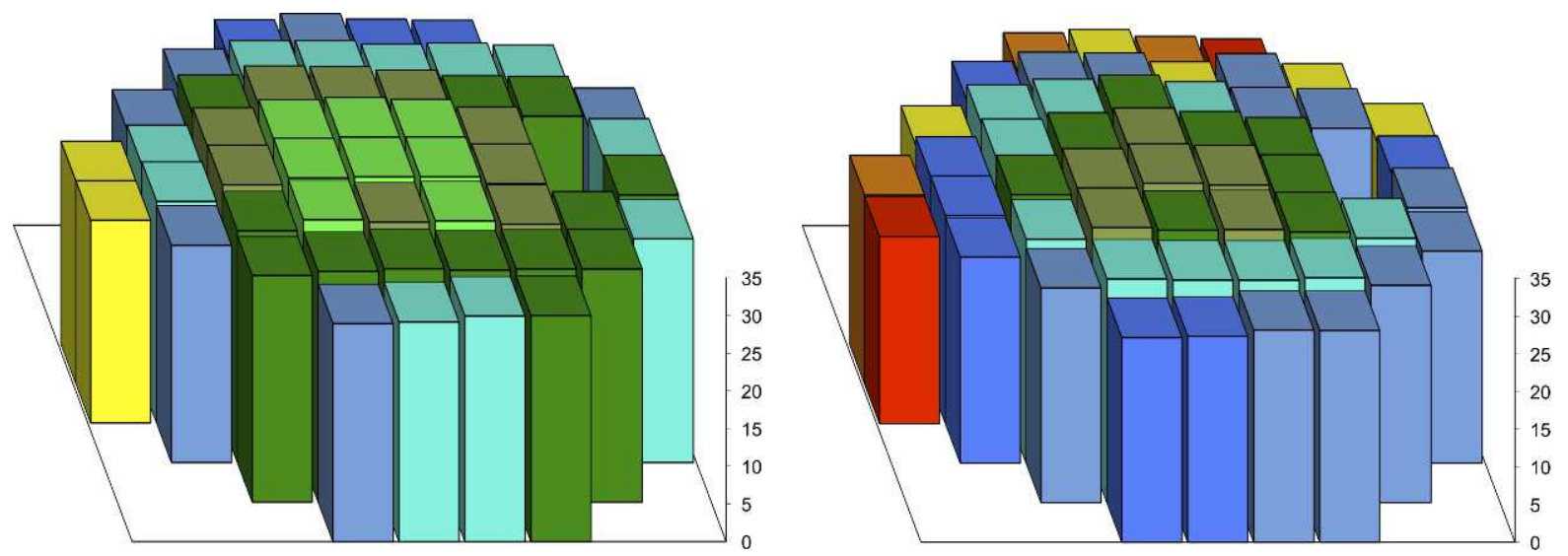

Figure 2. Mean SAP sensitivity threshold values (dB) in the control group (left graph) and the OSA group (right graph) for a right eye. The group with OSA had reduced sensitivity at most points tested by white-on-white perimetry compared with the control group, mainly in the peripheral visual field. 


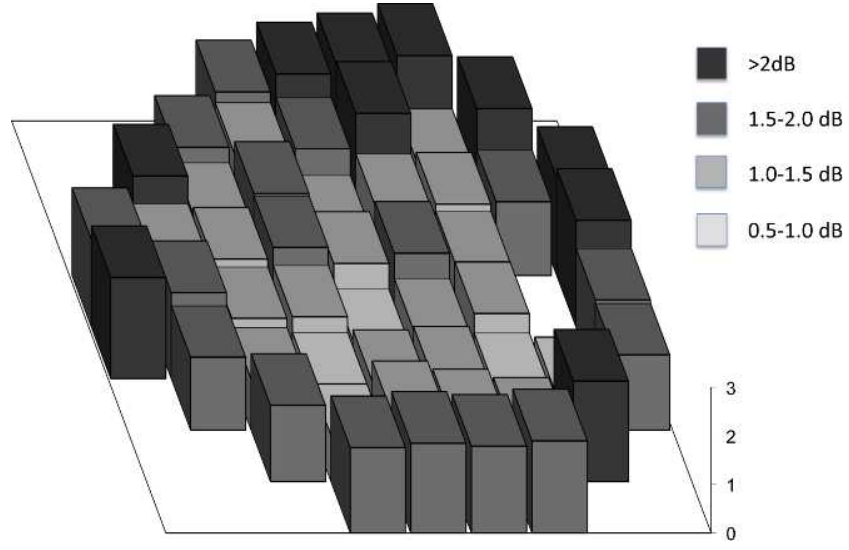

Figure 3. Differences in mean SAP sensitivity threshold values (dB) of the right eye between the control group and the OSA group. The grayscale represents the mean difference in decibels.

should have also undergone a polysomnographic evaluation for one night, but this evaluation is costly and we could not justify its inclusion in the study protocol because the criteria used to select the control group minimized the possibility of including an OSA patient by chance (exclusion criteria for the control group: obesity and symptoms related to OSA). Nevertheless, if all controls were chosen according to a strictly normal sleep study, we would have selected a "super-healthy" control group. In addition to the effect size (difference between groups), these factors would probably lead to a larger retinal sensitivity reduction than the one observed.

In the present study, OCT did not demonstrate neurodegenerative changes in OSA patients, but retinal sensitivity was significantly reduced in OSA patients compared with healthy subjects. Given the issues related with subjective perimetry, studies employing objective functional testing (e.g., electro-

TABLE 3. Differences in Peripapillary RNFL Thickness Measured by OCT Between the Control and OSA Groups

\begin{tabular}{|c|c|c|c|c|c|c|c|c|c|}
\hline & \multicolumn{4}{|c|}{ Control Group } & \multicolumn{4}{|c|}{ OSA Patients } & \multirow[b]{2}{*}{$\boldsymbol{P}^{*}$} \\
\hline & Min & Max & Mean & SD & Min & Max & Mean & SD & \\
\hline $\mathrm{H} 1$ & 58 & 164 & 108.86 & 21.62 & 30 & 163 & 110.24 & 22.52 & 0.67 \\
\hline $\mathrm{H} 2$ & 52 & 139 & 92.82 & 18.21 & 61 & 160 & 92.29 & 19.04 & 0.84 \\
\hline $\mathrm{H} 3$ & 40 & 83 & 62.22 & 9.89 & 45 & 89 & 60.30 & 8.82 & 0.16 \\
\hline $\mathrm{H} 4$ & 41 & 115 & 70.05 & 13.87 & 30 & 112 & 69.45 & 14.38 & 0.77 \\
\hline $\mathrm{H} 5$ & 61 & 162 & 107.18 & 21.74 & 77 & 210 & 112.58 & 27.07 & 0.13 \\
\hline H6 & 78 & 211 & 144.75 & 25.70 & 71 & 213 & 147.10 & 25.19 & 0.53 \\
\hline $\mathrm{H}_{7}$ & 81 & 207 & 140.91 & 22.84 & 92 & 181 & 135.13 & 20.15 & 0.07 \\
\hline H8 & 39 & 119 & 67.19 & 13.19 & 41 & 113 & 65.86 & 12.83 & 0.49 \\
\hline H9 & 34 & 73 & 50.40 & 7.77 & 36 & 88 & 52.74 & 7.72 & 0.04 \\
\hline $\mathrm{H} 10$ & 55 & 144 & 77.88 & 13.85 & 43 & 100 & 75.21 & 11.35 & 0.16 \\
\hline H11 & 90 & 184 & 133.41 & 17.63 & 10 & 709 & 133.30 & 69.77 & 0.99 \\
\hline H12 & 65 & 195 & 121.76 & 26.97 & 39 & 183 & 127.49 & 27.71 & 0.15 \\
\hline Superior & 93 & 169 & 121.39 & 15.65 & 55 & 155 & 120.93 & 17.16 & 0.86 \\
\hline Inferior & 42 & 165 & 130.04 & 17.13 & 96 & 171 & 131.11 & 15.68 & 0.66 \\
\hline Nasal & 38 & 110 & 74.90 & 11.61 & 52 & 148 & 76.06 & 15.38 & 0.55 \\
\hline Temporal & 47 & 104 & 65.12 & 9.55 & 30 & 88 & 64.49 & 8.70 & 0.64 \\
\hline \multicolumn{10}{|l|}{ Average } \\
\hline thickness & 80 & 120 & 98.04 & 8.21 & 61 & 120 & 97.15 & 9.71 & 0.50 \\
\hline$n$ & & & 111 & & & & 80 & & \\
\hline
\end{tabular}

H, RNFL thickness at every clock-hour position for a right eye; Min, minimum; Max, maximum.

* Student's $t$-test.
TABLE 4. Analysis of Peripapillary RNFL Thickness Measurements by OCT in Healthy Controls Versus OSA Grade 2 and 3 Patients

\begin{tabular}{|c|c|c|c|c|c|c|c|c|}
\hline & $\begin{array}{l}\text { Cont } \\
\text { Gro }\end{array}$ & $\begin{array}{l}\text { trol } \\
\text { up }\end{array}$ & $\begin{array}{r}\mathbf{G} \\
2 \\
\text { OSA }\end{array}$ & $\begin{array}{l}\text { rades } \\
\text { And } 3 \\
\text { Patien }\end{array}$ & & $\begin{array}{r}\text { Gr } \\
\text { OSA }\end{array}$ & $\begin{array}{l}\text { rade } 3 \\
\text { Patien }\end{array}$ & \\
\hline & Mean & SD & Mean & SD & $\boldsymbol{P}^{*}$ & Mean & SD & $P \dagger$ \\
\hline $\mathrm{H} 1$ & 108.86 & 21.62 & 111.16 & 20.67 & 0.48 & 110.52 & 20.32 & 0.64 \\
\hline $\mathrm{H} 2$ & 92.82 & 18.21 & 92.35 & 19.09 & 0.87 & 91.04 & 18.65 & 0.56 \\
\hline H3 & 62.22 & 9.89 & 59.87 & 8.87 & 0.22 & 59.71 & 8.13 & 0.22 \\
\hline H4 & 70.05 & 13.87 & 70.44 & 14.71 & 0.87 & 70.90 & 13.82 & 0.72 \\
\hline $\mathrm{H} 5$ & 107.18 & 21.74 & 109.57 & 24.45 & 0.50 & 104.75 & 21.69 & 0.51 \\
\hline H6 & 144.75 & 25.70 & 144.10 & 24.11 & 0.87 & 141.40 & 23.57 & 0.43 \\
\hline $\mathrm{H} 7$ & 140.91 & 22.84 & 136.57 & 20.12 & 0.20 & 136.77 & 18.84 & 0.26 \\
\hline H8 & 67.19 & 13.19 & 66.69 & 12.95 & 0.81 & 68.10 & 12.12 & 0.68 \\
\hline H9 & 50.40 & 7.77 & 53.09 & 8.18 & 0.03 & 53.75 & 8.50 & 0.01 \\
\hline H10 & 77.88 & 13.85 & 75.26 & 11.93 & 0.20 & 75.44 & 11.37 & 0.27 \\
\hline H11 & 133.41 & 17.63 & 135.34 & 75.16 & 0.80 & 136.25 & 85.53 & 0.74 \\
\hline H12 & 121.76 & 26.97 & 128.68 & 27.16 & 0.10 & 125.96 & 27.09 & 0.3 \\
\hline Superi & 121.39 & 15.65 & 121.87 & 16.43 & 0.85 & 120.38 & 16.80 & 0.71 \\
\hline Inferio & 130.04 & 17.13 & 129.51 & 15.51 & 0.84 & 127.52 & 14.74 & 0.36 \\
\hline Nasal & 74.90 & 11.61 & 76.51 & 16.06 & 0.44 & 73.52 & 11.58 & 0.48 \\
\hline Temporal & 65.12 & 9.55 & 65.41 & 8.06 & 0.83 & 66.23 & 7.87 & 0.46 \\
\hline $\begin{array}{l}\text { Average } \\
\text { thickness }\end{array}$ & 98.04 & 8.21 & 97.03 & 10.20 & 0.47 & 96.90 & 9.09 & 0.43 \\
\hline$n$ & 11 & & & 68 & & & 52 & \\
\hline
\end{tabular}

* Student's $t$-test between grade 2 and 3 OSA patients and the control group.

† Student's $t$-test between grade 3 OSA patients and the control group.

physiology test) are warranted, as well as further prospective longitudinal studies to clarify the role of VF and OCT as a neurodegenerative marker in OSA syndrome.

Clinicians should be aware that OSA patients may have nonglaucomatous VF defects, which should be differentiated from true glaucomatous optic neuropathy.

\section{Acknowledgments}

Supported in part by the Instituto de Salud Carlos III Grant PI1101239.

Disclosure: B. Ferrandez, None; A. Ferreras, None; P. Calvo, None; B. Abadia, None; P. Fogagnolo, None; Y. Wang, None; J.M. Marin, None; M. Iester, None

\section{References}

1. Duran J, Esnaola S, Rubio R, Iztueta A. Obstructive sleep apnea-hypopnea and related clinical features in a populationbased sample of subjects aged 30 to $70 \mathrm{yr}$. Am J Respir Crit Care Med. 2001;163:685-689.

2. Young T, Peppard P, Palta M, et al. Population-based study of sleep-disordered breathing as a risk factor for hypertension. Arch Intern Med. 1997;157:1746-1752.

3. Peker Y, Kraiczi H, Hedner J, et al. An independent association between obstructive sleep apnoea and coronary artery disease. Eur Respir J. 1999;14:179-184.

4. Tsai JC. Neurological and neurobehavioral sequelae of obstructive sleep apnea. NeuroRehabilitation. 2010;26:8594.

5. Zhang X, Ma L, Li S, Wang Y, Wang L. A functional MRI evaluation of frontal dysfunction in patients with severe obstructive sleep apnea. Sleep Med. 2011;12:335-340. 
6. Bajpai S, Im KB, Dyken ME, Sodhi SK, Fiedorowicz JG. Obstructive sleep apnea and risk for late-life depression. Ann Clin Psychiatry. 2014;26:E1-E8.

7. Aurora RN, Punjabi NM. Obstructive sleep apnoea and type 2 diabetes mellitus: a bidirectional association. Lancet Respir Med. 2013;1:329-338.

8. Nannapaneni S, Ramar K, Surani S. Effect of obstructive sleep apnea on type 2 diabetes mellitus: a comprehensive literature review. World J Diabetes. 2013;4:238-244.

9. Morgenstern M, Wang J, Beatty N, Batemarco T, Sica AL, Greenberg H. Obstructive sleep apnea: an unexpected cause of insulin resistance and diabetes. Endocrinol Metab Clin North Am. 2014;43:187-204.

10. Surani SR. Diabetes, sleep apnea, obesity and cardiovascular disease: Why not address them together? World J Diabetes. 2014;5:381-384.

11. Levy P, Tamisier R, Arnaud C, et al. Sleep deprivation, sleep apnea and cardiovascular diseases. Front Biosci (Elite Ed). 2012;4:2007-2021.

12. Gozal D, Kheirandish-Gozal L. Cardiovascular morbidity in obstructive sleep apnea: oxidative stress, inflammation, and much more. Am J Respir Crit Care Med. 2008;177:369-375.

13. Stoohs R, Guilleminault C. Cardiovascular changes associated with obstructive sleep apnea syndrome. J Appl Physiol. 1992; 72:583-589.

14. Sihota R, Sony P, Gupta V, et al. Diagnostic capability of optical coherence tomography in evaluating the degree of glaucomatous retinal nerve fiber damage. Invest Ophthalmol Vis Sci. 2006; 47:2006-2010.

15. Ferreras A, Pablo LE, Pajarín AB, Larrosa J, Polo V, Honrubia FM. Logistic regression analysis for early glaucoma diagnosis using optical coherence tomography. Archives Ophthalmol. 2008;126:465-470.

16. Mwanza JC, Oakley JD, Budenz DL, Anderson DR; Cirrus Optical Coherence Tomography Normative Database Study Group. Ability of cirrus HD-OCT optic nerve head parameters to discriminate normal from glaucomatous eyes. Ophthalmology. 2011;118:241-248.

17. Mwanza JC, Oakley JD, Budenz DL, Chang RT, Knight OJ, Feuer WJ. Macular ganglion cell-inner plexiform layer: automated detection and thickness reproducibility with spectral domain-optical coherence tomography in glaucoma. Invest Ophthalmol Vis Sci. 2011;52:8323-8329.

18. Hwang YH, Kim YY. Glaucoma diagnostic ability of quadrant and clock-hour neuroretinal rim assessment using cirrus HD optical coherence tomography. Invest Opbthalmol Vis Sci. 2012;53:2226-2234.

19. Hwang YH, Kim YY, Kim HK, Sohn YH. Ability of Cirrus highdefinition spectral-domain optical coherence tomography clock-hour, deviation, and thickness maps in detecting photographic retinal nerve fiber layer abnormalities. Ophthalmology. 2013;120:1380-1387.

20. Tian T, Zhu XH, Liu YH. Potential role of retina as a biomarker for progression of Parkinson's disease. Int J Ophthalmol. 2011;4:433-438.

21. Adam CR, Shrier E, Ding Y, Glazman S, Bodis-Wollner I. Correlation of inner retinal thickness evaluated by spectraldomain optical coherence tomography and contrast sensitivity in Parkinson disease. J Neuroophthalmol. 2013;33:137-142.

22. Fjeldstad AS, Carlson NG, Rose JW. Optical coherence tomography as a biomarker in multiple sclerosis. Expert Opin Med Diagn. 2012;6:593-604.

23. Fernandes DB, Raza AS, Nogueira RG, et al. Evaluation of inner retinal layers in patients with multiple sclerosis or neuromyelitis optica using optical coherence tomography. Ophthalmology. 2013;120:387-394.
24. Kesler A, Vakhapova V, Korczyn AD, Naftaliev E, Neudorfer M. Retinal thickness in patients with mild cognitive impairment and Alzheimer's disease. Clin Neurol Neurosurg. 2011;113: 523-526.

25. Marziani E, Pomati S, Ramolfo P, et al. Evaluation of retinal nerve fiber layer and ganglion cell layer thickness in Alzheimer's disease using spectral-domain optical coherence tomography. Invest Ophthalmol Vis Sci. 2013;54:5953-5958.

26. Berry RB, Budhiraja R, Gottlieb DJ, et al. Rules for scoring respiratory events in sleep: update of the 2007 AASM Manual for the Scoring of Sleep and Associated Events. Deliberations of the Sleep Apnea Definitions Task Force of the American Academy of Sleep Medicine. J Clin Sleep Med. 2012;8:597619.

27. Chylack LT Jr, Wolfe JK, Singer DM, et al. The Lens Opacities Classification System III. The Longitudinal Study of Cataract Study Group. Arch Ophthalmol. 1993;111:831-836.

28. Calvo P, Ferrández B, Ferreras A, Marín JM. Retinal nerve fiber layer thickness alterations in patients with obstructive sleep apnea. Arch Soc Esp Oftalmol. 2012;87:1-2.

29. Karaca Z, Ismailogullari $S$, Korkmaz $S$, et al. Obstructive sleep apnoea syndrome is associated with relative hypocortisolemia and decreased hypothalamo-pituitary-adrenal axis response to 1 and $250 \mu \mathrm{g} \mathrm{ACTH}$ and glucagon stimulation tests. Sleep Med. 2013;14:160-164.

30. Shahar E, Whitney CW, Redline S, et al. Sleep-disordered breathing and cardiovascular disease: crosssectional results of the Sleep Heart Health Study. Am J Respir Crit Care Med. 2001;163:19-25.

31. Anderson DR. Glaucoma, capillaries and pericytes. 1. Blood flow regulation. Ophthalmologica. 1996;210:257-262.

32. Calvo P, Ferreras A, Polo V, et al. Predictive value of retrobulbar blood flow velocities in glaucoma suspects. Invest Ophthalmol Vis Sci. 2012;53:3875-3884.

33. Lin CC, Hu CC, Ho JD, Chiu HW, Lin HC. Obstructive sleep apnea and increased risk of glaucoma: a population-based matched-cohort study. Ophthalmology. 2013;8:1559-1564.

34. Garway-Heath DF, Poinoosawmy D, Fitzke FW, Hitchings RA. Mapping the visual field to the optic disc in normal tension glaucoma eyes. Ophthalmology. 2000;107:1809-1815.

35. Ferreras A, Pablo LE, Garway-Heath DF, Fogagnolo P, GarcíaFeijoo J. Mapping standard automated perimetry to the peripapillary retinal nerve fiber layer in glaucoma. Invest Ophthalmol Vis Sci. 2008;49:3018-3025.

36. Lamparter J, Russell RA, Zhu $\mathrm{H}$, et al. The influence of intersubject variability in ocular anatomical variables on the mapping of retinal locations to the retinal nerve fiber layer and optic nerve head. Invest Ophthalmol Vis Sci. 2013;54:60746082.

37. Lopez-Pena MJ, Ferreras A, Polo V, et al. Relationship between standard automated perimetry and HRT, OCT and GDx in normal, ocular hypertensive and glaucomatous subjects. Arch Soc Esp Oftalmol. 2007;82:197-208.

38. Lopez-Pena MJ, Ferreras A, Larrosa JM, et al. Relationship between standard automated perimetry and retinal nerve fiber layer parameters obtained with optical coherence tomography. J Glaucoma. 2011;20:422-432.

39. Kargi SH, Altin R, Koksal M, et al. Retinal nerve fiber layer measurements are reduced in patients with obstructive sleep apnea syndrome. Eye. 2005;19:575-579.

40. Lin PW, Friedman M, Lin HC, Chang HW, Pulver TM, Chin CH. Decreased retinal nerve fiber layer thickness in patients with obstructive sleep apnea/hypopnea syndrome. Graefes Arch Clin Exp Ophthalmol. 2011;249:585-593.

41. Casas P, Ascaso FJ, Vicente E, Tejero-Garcés G, Adiego MI, Cristóbal JA. Retinal and optic nerve evaluation by optical coherence tomography in adults with obstructive sleep apnea- 
hypopnea syndrome (OSAHS). Graefes Arch Clin Exp Ophthalmol. 2013;251:1625-1634.

42. Moghimi S, Ahmadraji A, Sotoodeh $\mathrm{H}$, et al. Retinal nerve fiber thickness is reduced in sleep apnea syndrome. Sleep Med. 2013; 14:53-57.

43. Sagiv O, Fishelson-Arev T, Buckman G, et al. Retinal nerve fiber layer thickness measurements by optical coherence tomography in patients with sleep apnea syndrome. Clin Experiment Ophthalmol. 2014;42:132-138.
44. Huseyinoglu N, Ekinci M, Ozben S, Buyukuysal C, Kale MY, Sanivar HS. Optic disc and retinal nerve fiber layer parameters as indicators of neurodegenerative brain changes in patients with obstructive sleep apnea syndrome. Sleep Breath. 2014; 18:95-102.

45. Benbir G, Karadeniz D. A pilot study of the effects of noninvasive mechanical ventilation on the prognosis of ischemic cerebrovascular events in patients with obstructive sleep apnea syndrome. Neurol Sci. 2012;33:811-818. 